\title{
A solid state CFF apparatus
}

\author{
ALLAN H. FREY \\ Randomline, Inc., Huntingdon Valley, Pennsylvania 19006
}

In past critical fusion frequency (CFF) experiments, one of two methods for generating the flashing light has typically been used: a strobe light or a motor-driven sectored disk that chops the light from a lamp. Both of these methods generate noise that can cue a subject. A solid state apparatus has been designed to avoid this and other deficiencies of currently used methods. In use, the apparatus has been found to be simple, flexible, and reliable, and it does not give the subject acoustic cues to flicker rate.

In past critical fusion frequency (CFF) experiments, one of two methods for generating the flashing light has typically been used: a strobe light or a motor-driven sectored disk that chops the light from a lamp. Both of these methods generate noise that can cue a subject. A strobe light produces a clicking sound that is correlated with flicker rate. The sound of the motor driving the sectored disk is also correlated with the flicker rate. Further, the motor does not change speed instantly, which introduces an uncertainty in such CFF measurement.

A solid state apparatus has been designed to avoid the deficiencies noted above. The apparatus is described in the context of its three major assemblies: a lightemitting diode (LED), an integrated circuit (IC) assembly, and a rotary stepping switch assembly. These are shown in Figure 1.

The apparatus uses an LED as a light source or, if desired, several LEDs can be used in parallel to increase brightness. But a single LED is bright enough to be used under high ambient light conditions (e.g., an office).

The IC (LM 324) assembly generates square-wave pulses that cause the LED to flash. The IC contains four operational amplifiers, only one of which is shown as used in Figure 1. For many experiments, one is enough. The pulse rate is determined by the resistances of the set of potentiometers shown. For clarity, only six potentiometers are drawn, but many more can be used. The IC uses a 5-V power supply, as does the LED, which is supplied through the IC.

The relay $\left(M_{2}\right)$ switches the number one pin of the IC between $R_{10}$ and the common contact of the rotary stepping switch. $R_{10}$ sets the "steady" rate of the LED, that is, a pulse rate of 200 pulses/sec (pps). A dc voltage applied to the LED could provide a steady light, but it is too bright.

The normally open contact of $M_{2}$ is connected to the common contact of the rotary stepping relay $\left(M_{1}\right)$. The other contacts of $M_{1}$ are each connected to one of the remaining five potentiometers $\left(R_{5}-R_{10}\right)$, each of

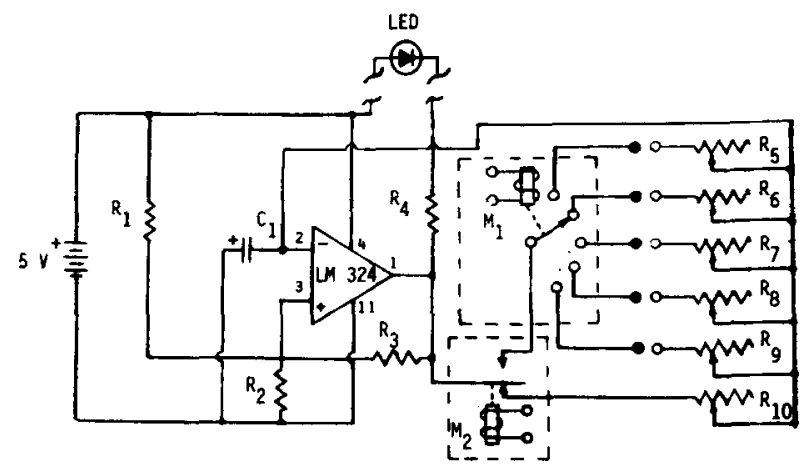

Figure 1. CFF apparatus circuit design. $R_{1}-R_{3}=100 \mathrm{kohm}$, $1 / 4 \mathrm{~W} ; \mathbf{R}_{4}=100 \mathrm{ohm}, 1 / 4 \mathrm{~W} ; \mathbf{R}_{5}-\mathbf{R}_{10}=$ megohm linear taper potentiometer, $1 / 4 \mathrm{~W} ; \mathrm{C}_{1}=.1$ microfarad, $50 \mathrm{WV} \mathrm{dc} ; \mathrm{M}_{1}=24-\mathrm{V}$ de rotary stepping switch; $M_{2}=24-V$ relay.

which sets a flicker rate as a function of its resistance. The stepping relay contacts are connected to the potentiometers through a barrier strip. After the potentiometers are set, the order of the flicker rates can be varied by changing the order of the connections on the barrier strip. The wipers of all six potentiometers are connected to Pin 2 of the IC. Pulse rates between approximately 10 and 200 pps can be set by adjustment of each potentiometer shown.

The coils of the relays $\left(\mathrm{M}_{1}\right.$ and $\left.\mathrm{M}_{2}\right)$ are driven by $24 \mathrm{~V}$ dc supplied by programming equipment that controls the experiment. If the stepping relay chosen is noisy when it steps to a new flash rate, it can be remotely located.

An alternate IC assembly can be constructed with the use of other ICs, such as Number 3909. Although the circuit is slightly simpler with the 3909 , the circuit requires capacitors to set the flicker rate. Capacitors cannot be readily adjusted as can potentiometers.

In use, the apparatus described has been found to be simple, flexible, and reliable, and it does not give the subject acoustic cues to the flicker rate.

(Accepted for publication October 15, 1979.) 